\title{
PARAMETRIC DESIGN ANALYSIS AND SHAPE OPTIMIZATION OF PERCUTANEOUS AORTIC VALVE STENTS
}

\author{
Gideon Praveen Kumar. ', Lazar Mathew ${ }^{2}$ \\ 1,2 Chemical and Biomedical Engineering, VIT University, Vellore, India \\ Email: gideonpraveenkumar@yahoo.com
}

\begin{abstract}
Vascular support structures are an important tool for treating valve stenosis. Alarge population of patients are treated for valvular disease and the principal mode of treatment is the use of percutaneous valvuloplasty. Stent devices are proving to be an improved treatment method; these devices now account for $20 \%$ of treatments in Europe. This new technology provides highly effective results at minimal cost and short duration of hospitalization. Accurate and reliable structural analysis provides essential information to the design team in an environment where in vivo experimentation is extremely expensive, or impossible. This paper describes the design of vascular support structure (stents), to provide designers with estimates of the critical parameters which are essential to restore the functions of the endothelium of the Aorta during and after implantation without injuring it.
\end{abstract}

KEYWORDS: Stent, Blood flow, Aortic valve stenosis, Modeling, Finite element analysis

\section{I.INTRODUCTION}

The treatment of stenotic valvular diseases consists of routine procedures in interventional cardiology. To date, the surgical approach is the only option to replace diseased cardiac valves. Recently it has been shown that stenosis of mitral, aortic or pulmonary valves can be treated by percutaneous valve replacement and has opened new perspectives on transcatheter placement of cardiac valves. Aortic valve replacement generally has been accomplished by using a cardiac surgical procedure whereas endovascular procedures for valve replacement may provide an alternative to cardiac surgery. Percutaneous transluminal procedures have substantial benefits from the standpoints of health and safety, as well as cost.

Such endovascular procedures require minimal invasion of the human body, and there is considerable reduction and, in some instances, even elimination of general anesthesia and intensive care unit stay. With this view our team has attempted to generate models to achieve proper stenting and placement of the prosthesis without migration, paravalvular leaks and obstruction of coronary blood flow.

In percutaneous replacement, as shown in Fig 1 the diseased valve is replaced by a biological valve (porcine) via a catheter driven through the femoral artery to the aortic position. The valve is mounted on the stent, which is crimped into the catheter and is guided to the parent position of the aorta and deployed.

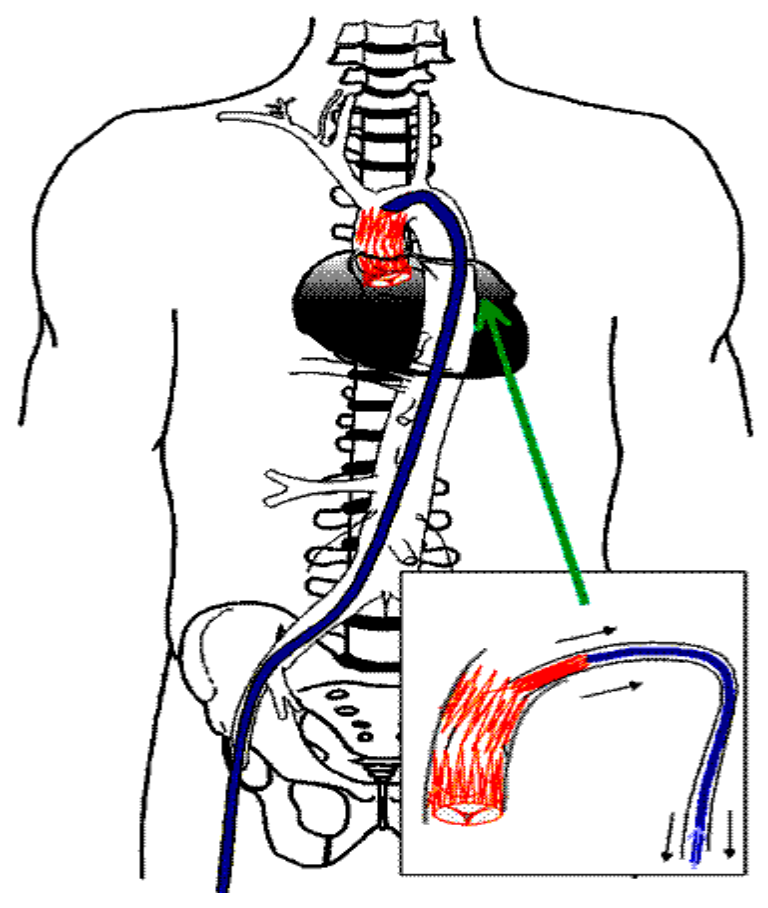

Fig. 1. Percutaneous Aortic Valve Replacement

Thus it pushes the diseased valve aside and keeps the new bio-prosthetic valve in position. Post operative complications are very minimal and the patient can get discharged within days after the procedure. The stents that are used give adequate support and stability to the valve and also prevent the prosthesis from getting migrated either in the ante grade or retro grade direction. These stents also help in preventing paravalvular leaks. With this view, we have generated novel models to achieve proper stenting and placement of the prosthesis without migration, paravalvular leaks and obstruction of coronary blood flow. 


\section{METHODS}

\section{A. Parametric Stent Design}

Stent geometies were uniquely defined using the following parameters (a)Diamter of the aorta (b) Distance between the aortic root and the coronary artery roots (c) Position of the coronary arteries (d) Diameter of the coronaries (e) Stent-Endothelium Mechanics. Keeping these parameters into consideration many stent models [Fig.2(a)-(c)]were designed to suit its requirement for percutaneous replacement.

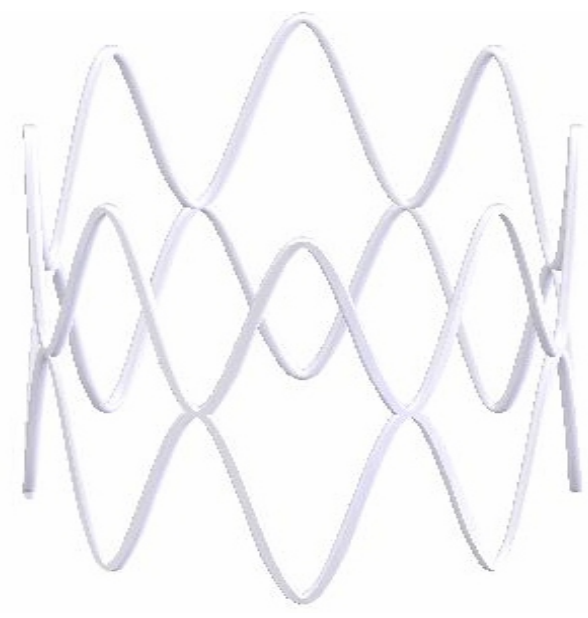

Fig. 2 (a) Model 1

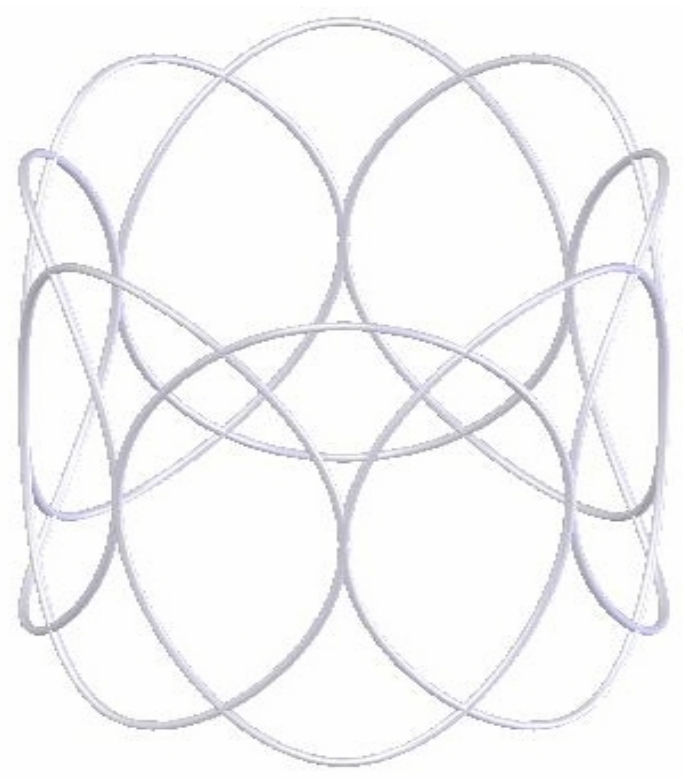

(b)Model 2

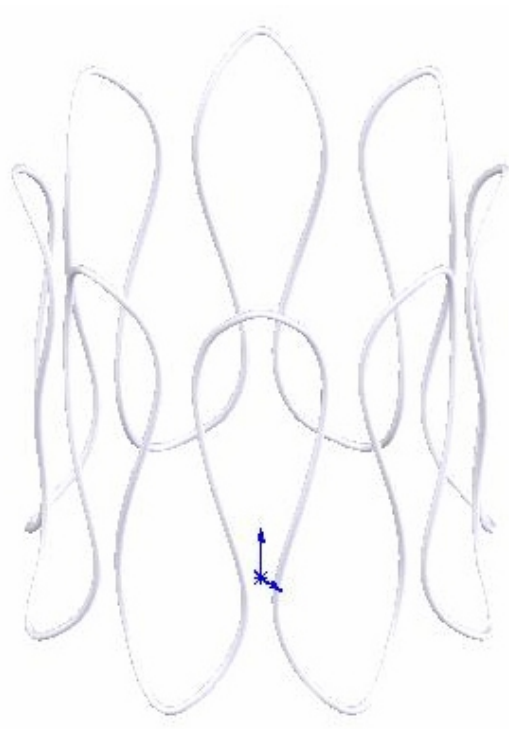

(c) Model 3

\section{B. Model Geometry}

Apart form these models another new model was designed. The 3D geometry of the repeaTable- units of the stent was generated using SOLIDWORKS modeling Software. Using the repeating unit geometry of each stent design, solid models were generated. The unit consisted of 8 lips with two non crossing struts making a circular diameter of $16 \mathrm{~mm}$ at the center and $18 \mathrm{~mm}$ at the top and bottom as shown in Fig 3 . This varying diameter, as shown at different parts of the stent creates the blunt hooks at either end of the stent. The port angle is 45 degrees with the two struts joining to form the circular stent. The stent design has a constant strut thickness of $0.5 \mathrm{~mm}$ and a height of $18 \mathrm{~mm}$ [7].

\section{Material Model}

Stainless steel $316 \mathrm{~L}$, is considered as the material for all the stent models. A bilinear, elasto-plastic model with isotropic hardening, Young's modulus $E=201 \mathrm{GPa}$, Poisson's ratio 0.3 , and Yield stress $170 \mathrm{MPa}$ was considered

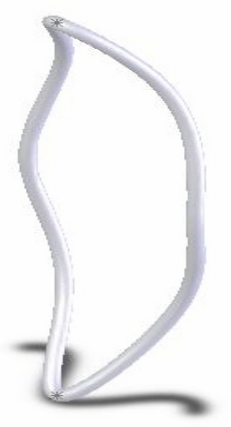

Fig. 3 (a) Single lip with 2 struts of $0.5 \mathrm{~mm}$ diameter 


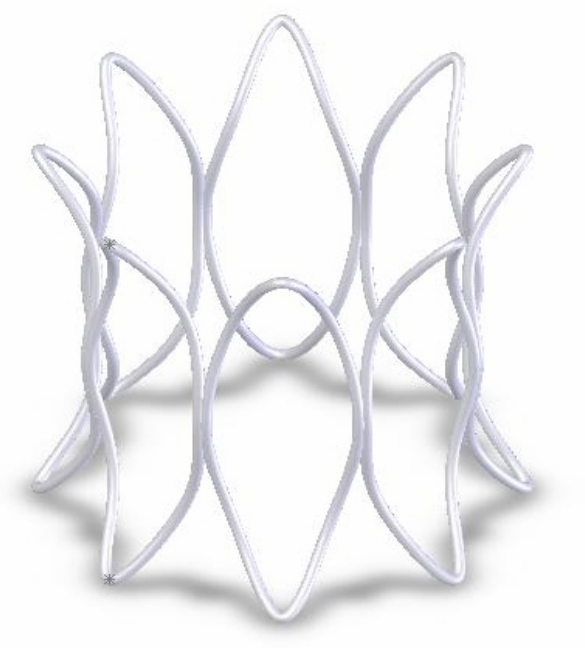

(b) The complete stent model having 8 lips

\section{Method of Analysis}

Free expansion analysis was done for the stent model using ANSYS finite element software. Mechanical properties like crimping and re-expansion were studied. The models were subjected to finite element analysis with boundary conditions . The stent solid model was then meshed to form a cylindrical shape by transferring the nodal co-ordinates from a Cartesian co-ordinate system into a cylindrical co-ordinate system. In this way Finite element meshes were generated for the design above the normal human blood pressure levels of $120 / 80 \mathrm{~mm} \mathrm{Hg}$ (as shown in Fig 4). The stent model seemed to withstand loads much more than that of the normal human pressure loads. However more analyses are done to study other mechanical properties like longitudinal recoil, foreshortening and to compare between the existing models and the proposed model.

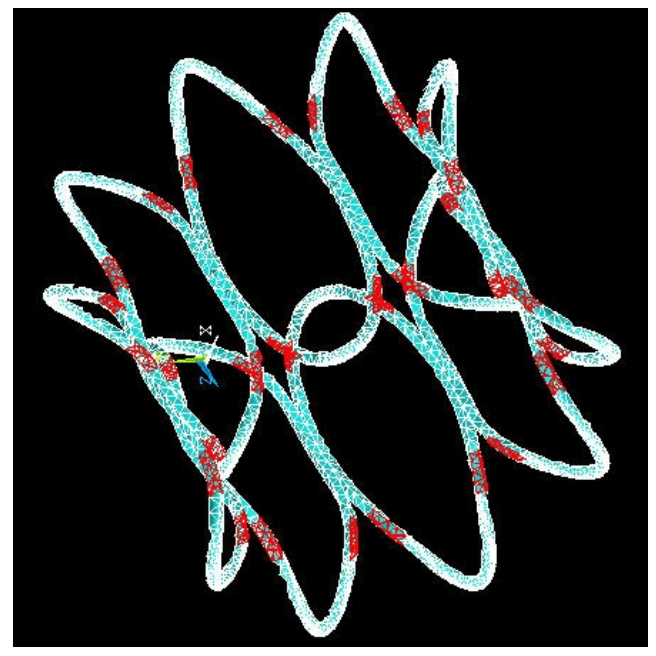

Fig. 4 (a) Load applied at key points

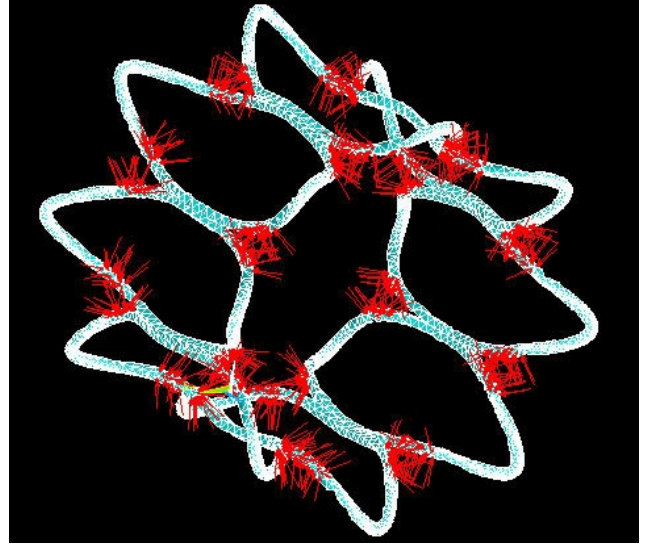

(b) Direction of load applied

\section{E. Fabrication}

A metal prototype (Model 3) was developed [Fig 5] to assess the performance of the model and the material selected. The wires are made of highly malleable $316 \mathrm{~L}$ Stainless Steel $0.5 \mathrm{~mm}$ cross section. This radially expanding vascular stent $(18 \mathrm{~mm}$ in length and $16 \mathrm{~mm}$ in diameter) includes several cylindrical fine-wire sections that are interconnected into a single tubular structure. The final two ends are welded using spot to spot stainless steel welding

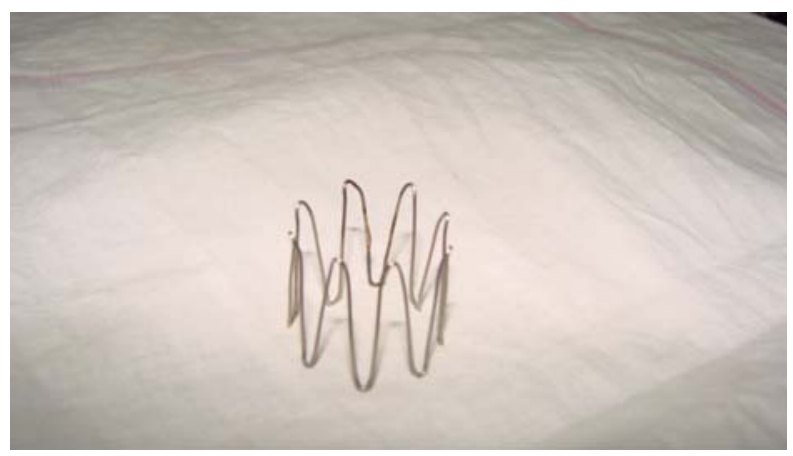

Fig. $50.5 \mathrm{~mm}$ Stainless Steel Prototype

\section{RESULTS AND DISCUSSION}

The stent models were designed and analyzed successfully. It was inferred that this stent designed carried some merits which its predecessors did not. Note the length of the model. The dimension of the stent, such as the length can influence the deliverability, visibility and flexibility of the stent. "Work at Thoraxcentre has clearly demonstrated that re-stenosis rates are predicTable- from stent and vessel dimensions." In their clinical study, the data clearly showed that short stents have lower cases of re-stenosis than long stent. Moreover the stent being too long, there are chances for tissue overgrowth around the stent. Strut geometry is another important engineering aspect. A study in rabbit iliac arteries indicated that less struts induce less chance of re-stenosis compare to more 
struts. That is the reason why we have come out with a design having 8 lips have 2 struts each given adequate gap between two adjacent struts. This gap between struts will enable the surgeon to place the stent in such a way that it doesn't obstruct the coronary blood flow which is situated just above the aortic valve. Another note worthy stent geometry is the cross-section of the strut. We have preferred round struts over square struts. Round strut cross-section area is ideal for smoothness design. On the other hand, squared strut cross-section area in not recommend because it interferes with blood flow due to their sharp edge which can slice blood cells.. Furthermore the blunt hooks that are designed help the stent to take its position once it is implanted by the surgeon. The aorta takes the shape of the stent one these blunt hooks get embedded into the endothelium of the aorta. This gives the stent a good fit into the aorta preventing it from getting migrated in either direction. This is a novel phenomenon which happens in this stent unlike the injuries caused by the barbed hooks that are seen in the already existing stents. These barbed hooks are intended to clasp the aorta in order to prevent migration

\section{CONCLUSION}

The stent models were designed and analyzed successfully. Methodology described in this paper is proposed as a method to compare and analyze the existing stents and the ones proposed here. However further analysis and studies are needed before these stents are fabricated and deployed. Animal experiments are being planned currently for this purpose

\section{ACKNOWLEDGEMENT}

We would like to thank Dr. Mohan Thanikachalam, Cardiac Surgeon, Christian Medical College and Hospital for giving us valuable suggestions whenever necessary. We would like to thank L-Ramp for funding our preliminary research. We would like to thank VIT University for allowing us to use the resources available to carry out our research.

\section{REFERENCES}

[1] Morton Allison C, Crossman. D, Gunn. J, The influence of physical stent parameters upon restenosis, Elsevier SAS, 2004.

[2] Lau. KW, Johan A, Sigwart U, Hung JS, A stent is not just a stent: stent construction and design do matter in its clinical performance, Singapore Medical Journal, 2004.

[3] McClean Dougal R, MD, Eigler N, MD, Stent Design: Implications for Restenosis, MedReviews, LLC 2002

[4] Stent design properties and deployment ratio influence indexes of wall shear stress: a threedimensional computational fluid dynamics investigation within a normal artery : J Appl Physiol 97: 424-430, 2004.

[5] An overview of superelastic stent design: Min Invas Ther \& Allied Technol 2000: 9(3/4) 235-246

[6] Engineering aspects of stents design and their translation into clinical practice: Ann Ist super sAnltà 2007 |Vol. 43, no. 1: 89-100

[7] To stent or not to stent aortic dissection:good news for a chosen few, but who? European Heart Journal (2005) 26, 431-432, doi:10.1093/eurheartj/ehi119

[8] J. Q. Zhou, A. F. Corno, C. H. Huber, P. Tozzi, and L. K. von Segesser Self-expandable valved stent of large size: off-bypass implantation in pulmonary position Eur. J. Cardiothorac. Surg., August 1, 2003; 24(2): $212-216$

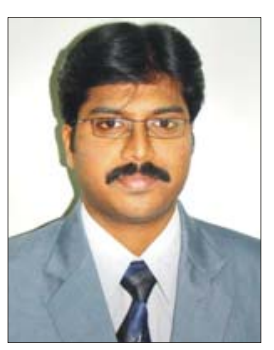

Mr. Gideon Praveen Kumar is a research scholar under the guidance of Dr.Lazar Mathew at the School of Biotechnology, Chemical and Biomedical Engineering, VIT University. His areas of specialization include CAD based design and finite element analysis of cardiovascular implants with special reference to percutaneous valve stents 